\title{
An Efficient End-to-End QoS Supporting Algorithm in NGN Using Optimal Flows and Measurement Feed-Back for Ubiquitous and Distributed Applications
}

\author{
Se Youn Ban ${ }^{1}$, Seong Gon $\mathrm{Choi}^{2}$, and Jun Kyun $\mathrm{Choi}^{1}$ \\ ${ }^{1}$ Information and Communication University \\ ${ }^{2}$ ChungBuk National University \\ syban@icu.ac.kr, sgchoi@chungbuk.ac.kr, jkchoi@icu.ac.kr
}

\begin{abstract}
This paper proposes an efficient end-to-end QoS supporting mechanism in Next Generation Network (NGN) with heterogeneous QoS capability to support ubiquitous and distributed applications. To address this, there should be proper admission control mechanism and adaptive provisioning to sustain endto-end QoS and maximize network utilization. Resource and Admission Control Subsystem of NGN has unique feature of centralized network resource control rather than other network control protocol system in IP network. By showing optimal flows in heterogeneous QoS capability network, NGN can support average delay of end-to-end which the applications could receive proper Quality of Service over network.
\end{abstract}

Keywords: NGN, end-to-end QoS, resource admission and control, differential service.

\section{Introduction}

Today's hottest issue is a Next Generation Network (NGN) to provide users consistent and ubiquitous services. The NGN discussed in ITU-T has such characteristics; packet-based transfer, broadband capabilities with end-to-end services, interworking with legacy networks, converged services between fixed and mobile network, and so on [1].

There are various data transport network with own QoS supporting technologies. Diffserv IP network shall be core network of NGN and the network resource shall be controlled by RACS in NGN [2]. Even though core network supports Diffserv, there are many different access network technologies which are usually layer 2 . Therefore we need end-to-end QoS supporting mechanisms for user applications in heterogeneous network environment where various layer 2 QoS technologies in access network and Diffserv QoS capability in core network coexist.

Three QoS provisioning mechanisms consist of classification, admission and reservation [9]. IntServ and DiffServ are main models to provide end-to-end QoS. RSVP [4] of IntServ guarantees absolute QoS for end-to-end QoS by per-flow basis reservation but it is very complex and there is scalability problem for all network 
equipments to understand it and support QoS. DiffServ model is scalable and easy to interwork with other QoS technologies but it provides relative QoS. Absolute DiffServ [8] is considered to give each class absolute QoS by aggregate basis reservation. It needs admission control schemes to guarantee absolute bound of specific QoS parameters. Admission control can be planning-based or measuring-based. Utilization-based admission control [9] based on planning is simpler and more scalable than RSVP [4] based on probing but it only concerns homogeneous Diffserv network.

Applications want network QoS based on IP 5-tuple flow for their services. NGN core network has Diffserv ability and Edge nodes between core and access network have per-flow control capability. Access Networks consist of various layer 2 technologies such as Ethernet, WirelessLAN, ADSL and so on. Theses layer 2 technologies have lack of supporting QoS of per-flow level. RACS of NGN tries to control and monitor all network equipments with assuming at least minimum interfaces between RACS and the equipments. The most of these mechanisms assume pure Diffserv network and under NGN environment, we need a QoS-supporting mechanism over heterogeneous networks which could not have Diffserv capability and RACS controllability.

Admission control is the most efficient way to protect core network to keep its ability to support end-to-end QoS. We can treat core network as Diffserv network and access networks as best effort network because data network technologies can not differentiate IP 5-tuple based flows. As RACS knows network topology, we can calculate optimal Diffserv flows among access and core network with heterogeneous environment. Edge nodes can perform admission control with pre-calculated optimal flows map. It can guarantee absolute QoS even in heterogeneous network. Also by changing classification of flows with class of Diffserv based on run-time measurement, it can maximize network utilization.

This paper consists of the following; Section 2 describes the related works in admission control mechanism in packet network. Section 3 proposes an architecture and algorithm of admission control to support end-to-end QoS with RACS of NGN and Section 4 shows numerical analysis. Finally, we conclude in section 5.

\section{Related Works}

Admission control in IP network can be categorized by two areas; Planning-based admission control like utilization-based admission control [3] uses arbitrary thresholds as admission control parameter. It is very simple and commonly used, but not easy to find optimal threshold to restrict traffic. Another approach in admission control is measuring-based admission control like RSVP[4] and agent-based selection[5]. It is efficient of using network resource and dynamically adapted to any network situation, but very complex and overhead because it sends a probe in network or measure every user request over core network. In this paper, we propose finding optimal threshold to maximize network utilization and adaptive QoS provisioning for end-to-end QoS based on QoS measurement. 


\subsection{Measurement-Based Admission Control [10]}

Measurement-based admission control algorithms (MBAC) were designed to achieve high levels of network utilization for such relaxed real-time services in IntServ model. The conservative worst-case parameter-based methods are wasting network resource to low utilization and hard to find even worst-case parameters. The MBAC for controlled-load service uses measurement to estimate existing traffic load and admits a new flow of rate $r$ if the following test succeeds of Measured Sum, $\hat{v} \prec v \mu-r$ where $\hat{v}$ is the measured load of existing traffic, and $v$ is a user-defined utilization target intended to limit the maximum link load. Upon admission of the new flow the load estimate is increased by $\hat{v}=\hat{v}+r$. It calculates the equivalent bandwidth for a set of flowing using the Hoeffding bounds.

The MBAC uses three measurement mechanisms: Time-window, Point Sampling and Exponential Averaging. Time-window measurement mechanism is to measure network load and used for the Measured Sum of admission control. It computes an average load every $\mathrm{S}$ sampling period. At the end of a measurement window $\mathrm{T}$, it uses the highest average from the just ended $\mathrm{T}$ as the load estimate for the next $\mathrm{T}$ window. When a new flow is admitted to the network, the estimate is increased by the rate of the new flow. If a newly computed average is above the estimate, the estimate is immediately raised to the new average. At the end of every $\mathrm{T}$, the estimate is adjusted to the actual load measured in the previous T. Point Sampling mechanism is the measurement mechanism which the sampling time window is in T/S ratio of 1 . Hoeffding bounds algorithm of MBAC uses Exponential Averaging mechanism to get an exponential average load. An average load is measured once every S sampling period. The exponential average load is computed using aninfinite impulse response (IIR) function

The MBAC allows for the greatest degree of flexibility in operation by relying on historical data for the tuning of its parameters. But in the view point of end-to-end differential service, it is hard to measure all user flows over network because it is studied in IntServ model and find optimal upper utilization parameters because it does not concern multi-node network.

\subsection{Utilization-Based Admission Control [9]}

Utilization-based Admission Control (UBAC) provides statistical guarantees within the diffserv model in a network, that uses static-priority schedulers. It derives delay bounds without specific information on flow population to employ a utilization-based admission control approach for flow admission. The UBAC does not require explicit delay computation at admission time and hence is scalable to large systems. In Integrated Services, connections are controlled both by admission control at connection establishment time and packet scheduling during the lifetime of the connections.

The mechanism of UBAC defines a utilization bound below which all the workload using the resource is guaranteed to meet its deadline. UBAC was first proposed in [22] for preemptive scheduling of periodic tasks on a single server. This idea was used in general multi-node networks in the diffserv setting for providing deterministic guarantees. It has assumptions about network nodes and network traffic follow the Differentiated Services architecture: In the network, they differentiates flow-aware 
edge routers from core routers, which are only aware of aggregations of flows in form of flow classes. The network traffic consists of flows. They limits our solution to the system with two flow classes. Each flow belongs to one of two flow classes: (1) highpriority class with deadline constraints, i.e., real-time class and (2) a low priority, best-effort class.

The UBAC consists of three mechanisms; Statistical flow-population-insensitive delay computation and utilization bound verification, Efficient admission control and Packet forwarding control. In configuration time, The UBAC estimates the delay upper bound for every class at each router with Statistical flow-population-insensitive delay computation and utilization bound verification using the flow populationinsensitive delay method. This verifies whether the end-to-end delay bound in each feasible path of the network satisfies the deadline requirement as long as the bandwidth usage on the path is within a pre-defined limit, i.e., the utilization bound. As the delay has been verified at configuration time, Efficient admission control mechanism can check only if the bandwidth is available along the path of the new flow. In packet forwarding control mechanism, routers transmits packets according to their priorities which are marked in their header. Within the same priority, packets are served in FIFO order.

The UBAC gives good theoretical background of efficient admission control in Diffserv model to support end-to-end QoS with delay bound. If the network is not defined as one Diffserv model, the UBAC does not apply its mechanism directly. In next chapter, this paper gives idea of how to get utilization bound with give class delay in heterogenous network with DiffServ and best-effort model and reduce the measurement overhead to sustain end-to-end QoS.

\section{Architecture and Mechanism of Admission Control to Support End-to-End QoS in NGN}

NGN network consists of two parts; access network and core network. There are many kinds of access networks which support network layer 2 technologies. They can support QoS for network layer 3. But many ubiquitous and distributed applications want end-to-end QoS of network layer 4; IP 5-tuple based QoS. NGN assumes that IP Diffserv and MPLS Diffserv are supported in core network [6]. RACS of NGN is one of solutions to supports resource and admission control to ensure end-to-end network resource for session service of applications. A problem of RACS is that when it provides like session service, it should check and handle network resource for each session service. This situation causes overhead in core network and access network, because all of service request from each access network flows into a core network and access network generally does not handle IP 5-tuple based flows. The rate to check and handle network resource in core network is much bigger than in access network and it is necessary to reduce complexity in core network. Edge Router (ER) is a gateway between core and access network. The admission control mechanism is located in ER.

Generally, various access networks (ex,ADSL,LAN...) do not support IP 5-tuple based QoS and they look like best-effort network in the point view of transport 


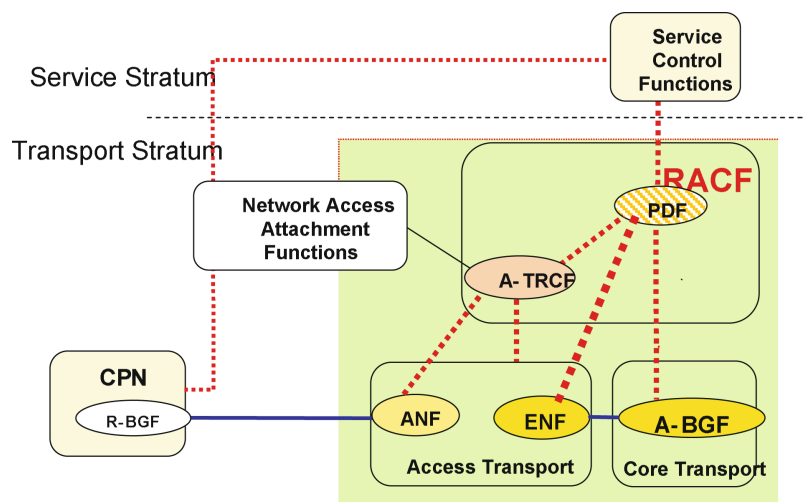

Fig. 1. Network Architecture of NGN

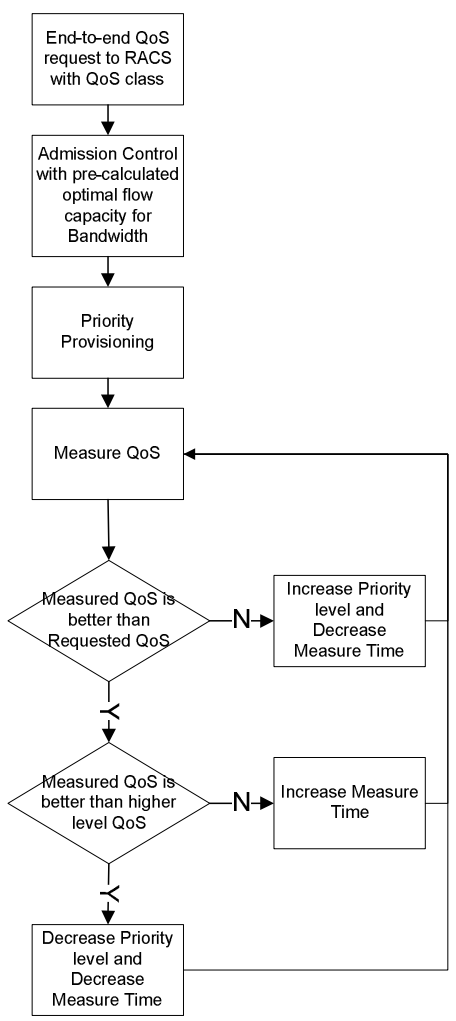

Fig. 2. Hop-based proportional Diffserv priority provisioning

network. NGN core network supports differential service and edge routers have perflow control capability. Of course, per-flow control capability can be in access nodes in access networks but cost and complexity are so high. 
The algorithm has two parts of provisioning and feedback. First, it processes admission and network resource provisioning. Second, it modifies the provisioning based on performance measurement. Because network situation is changing, the provisioning could change to satisfy end-to-end QoS. Additionally, because measureing performance of all flow is so complex, the algorithm also gives the way to reduce measurement of flow and period. It can extend the interval of measurement until the request QoS is not satisfied. Let's assume that user want real-time session service such like video conference. User requests Service Control Function (SCF) to support his service. SCF request Policy Decision Function (PDF) of RACS with bandwidth and QoS class. PDF processes network resource provisioning for end-to-end QoS. It can decide admission control of the request flow and give priority to guarantee the request QoS. After provisioning, it needs to confirm the service request satisfied by measuring performance. If measured QoS is worse than the requested QoS, ascend priority and decrease measure interval. If measured QoS shows lower priority could satisfy the request QoS, descend priority and decrease measure interval. If measured QoS shows the requested QoS is satisfied, increase measure interval.

\section{Numerical Analysis}

Let's think about [Fig 6] network architecture. Access network is network layer 2 network and its topology is based on tree architecture. Of course it does not need any routing capability and care IP 5-tuple flows. We assume the access network is besteffort network. Core network has differential service (Diffserv) capability. Edge Router of ingress node has per-flow control capability to classify, mark and admit packets. We assume the core network is Diffserv network with priority queueing.

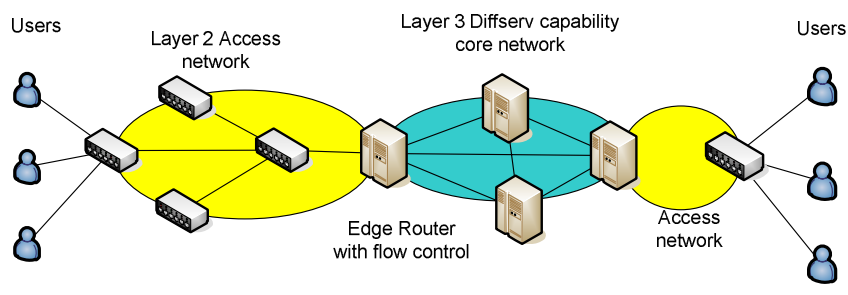

Fig. 3. Access and core network of different flow QoS capabilities

Diffserv network in real world is so complicate and there is a little proper formula to provide total average delay. So I consider Priority Queueing service to bound delay to satisfy quality of priority class and modify Optimal Flow Control Problem with packet network. Final objectives is to find optimal rate of priority class and hops to satisfy priority class delay bound even if it gives lower priority. In this formulation, I try to find optimal rate of priority class in simple case and the later will remain a future study.

Priority queuing model is one node analysis to find average delay and throughput with priority class. There are three kinds of Priority Discipline; Non-preemptive, 
Preemptive resume and Preemptive non-resume. Non-preemtive is used to formulate the problem.

Average waiting time for priority class $\mathrm{p}$ is below:

$E\left[W_{p}\right]=E\left[T_{0}\right]+\sum_{k=1}^{p} E\left[T_{k}\right]+\sum_{k=1}^{p-1} E\left[T_{k}^{\prime}\right]$

where $T_{0}$ : the completion time of current service

$T_{k}$ : service time of $m_{k}$ messages of priority $1,2, \ldots, p$ already waiting

$T_{k}^{\prime}$ : service time of $k=1,2, \ldots, p-1$ high priority message during the waiting time

$E\left[W_{p}\right]=E\left[T_{0}\right]+\sum_{k=1}^{p} E\left[T_{k}\right]+\sum_{k=1}^{p-1} E\left[T_{k}^{\prime}\right]=E\left[T_{0}\right]+\sum_{k=1}^{p} \rho_{k} E\left[W_{k}\right]+E\left[W_{p}\right] \sum_{k=1}^{p-1} \rho_{k}$ $E\left[W_{p}\right]=\frac{E\left[T_{0}\right]}{\left(1-\sigma_{p-1}\right)\left(1-\sigma_{p}\right)} \quad$ where $\sigma_{p}=\sum_{k=1}^{p} \rho_{k}$

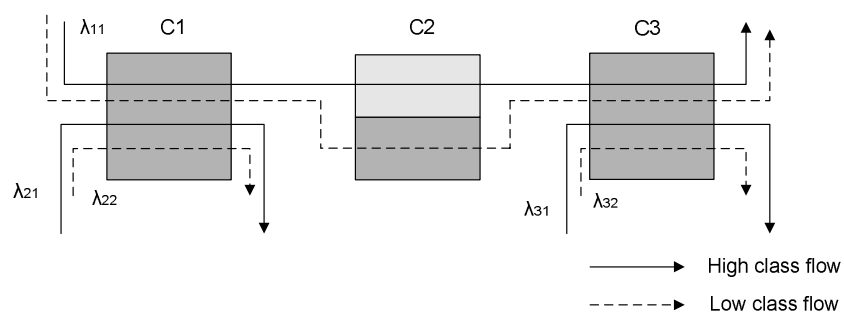

Fig. 4. Flows with two best-effort and one DiffServ network

Let's assume other situations:

1. Two priority classes, High priority - average delay bound to 100msec,Low priority - no average delay bound

2. nodes can accept traffic within max arrival rate

3. Core node has Non-preemptive priority queueing capability

4. Fixed Routing - Three flow paths

5. Processing (departure) rate of node is fixed

6. Average delay at node follows queueing model

7. No link delay $(0.3 \mathrm{msec}$ link delay per $100 \mathrm{~km}$ in real world)

And we want to maximize network utilization subjected to sum of arrival rates in a node is bound to max arrival capacity and sum of average delay of priority flow is bound.

$$
\begin{array}{ll}
\text { Maximize: } \quad & \sum_{\text {high }} U_{\text {high }}\left(\lambda_{i}\right)+\sum_{\text {low }} U_{\text {low }}\left(\lambda_{i}\right) \\
\text { Subject to: } \quad & \sum_{\text {ind } \lambda_{i}} \lambda_{i} \lambda_{\max } \\
& \sum_{\text {DiffServ_node }} \frac{\left(\rho_{1} / \mu_{1}\right)+\left(\rho_{2} / \mu_{2}\right)}{\left(1-\rho_{1}\right)}+\sum_{\text {BestEffort_node }} \frac{(\rho / \mu)}{(1-\rho)} \leq D_{\text {proirity }}
\end{array}
$$


Let's utilization function follows logarithm, High class is $30 \%$ more utility than low class and Utility is directly proportional to the number of hops of flow. Network supports IP class 0 QoS [7] and capacities of network are $10 \mathrm{Mbps}$.

$$
\begin{aligned}
& \text { Maximize: } \quad 3.9 \ln \left(\lambda_{11}\right)+1.3 \ln \left(\lambda_{21}\right)+1.3 \ln \left(\lambda_{31}\right)+3 \ln \left(\lambda_{12}\right)+\ln \left(\lambda_{22}\right)+\ln \left(\lambda_{32}\right) \\
& \text { Subject to: } \quad \lambda_{11}+\lambda_{21}+\lambda_{12}+\lambda_{22} \leq 10 M \\
& \lambda_{11}+\lambda_{12} \leq 10 M \\
& \lambda_{11}+\lambda_{31}+\lambda_{12}+\lambda_{32} \leq 10 M \\
& y_{1}+y_{2}+y_{3} \leq 0.1 \\
& \begin{array}{|l|l|l}
\frac{\lambda_{11}+\lambda_{12}}{1-\frac{\lambda_{11}}{10 M}} \leq y_{1} & \frac{\lambda_{11}+\lambda_{12}+\lambda_{21}+\lambda_{22}}{1-\frac{\lambda_{11}+\lambda_{12}+\lambda_{21}+\lambda_{22}}{10 M}} \leq y_{2} & \frac{\lambda_{11}+\lambda_{12}+\lambda_{31}+\lambda_{32}}{1-\frac{\lambda_{11}+\lambda_{12}+\lambda_{31}+\lambda_{32}}{10 M}} \leq y_{3}
\end{array}
\end{aligned}
$$

With this optimization problem, we can find the following result.

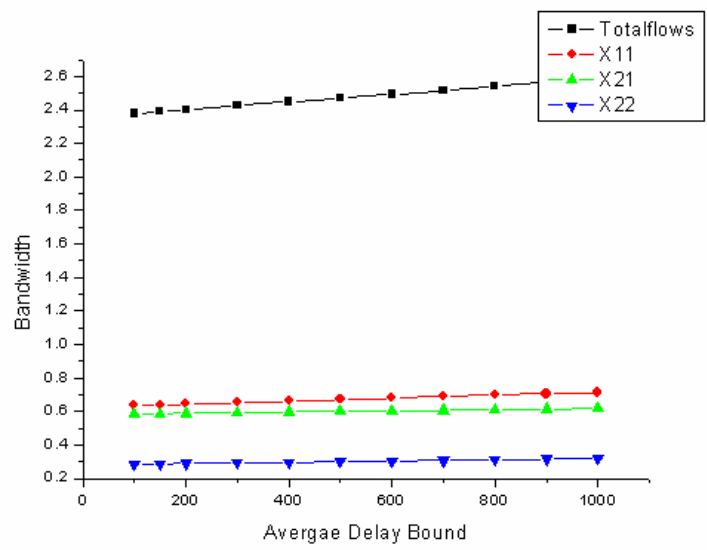

Fig. 5. Optimal flow with delay bound

Because total network capacity is 10 , this network utilization is about $25 \%$. Max utilization of best-effort queueing network is about 60\%.In heterogeneous QoS environment, it must use a third of network utilization to provide differential service and guarantee average delay bound for high QoS class. Of course, as average delay bound looses, the network utilization increases. If it increases average delay bound infinite, it is removing the delay bound constraint. The sum of optimal flow rate at node is as same as maximum flow rate capacity at node. It shows co-relationship between rate capacity and the saturation point and it means we can calculate node capacity given delay bound in Diffserv network vice versa.

As we see the result, long path flow causes network performance low. We can find the optimal flow rate given delay-bound and node capacity. If we know the optimal flow rate, we can use these pre-calculated optimal flows for admission control and modify Diffserv traffic with the optimal flow rate - like increasing or decreasing high priority flow to the optimal flow. We show the optimal flow and our algorithm is useful to optimize network utility. 


\section{Conclusion and Future Works}

In this paper, we have present category of admission control in IP network in NGN, architecture and algorithm of efficient admission control using pre-calculated optimal flows and measurement feed-back mechanism. The admission control based on optimal flows can guarantee end-to-end QoS among heterogeneous QoS capabilities of access networks and core network. Measurement feed-back mechanism can sustain the request QoS, reduce network overhead and maximize network utilization. We need to verify the algorithm for specific access networks and core network such as Ethernet and MPLS. Furthermore, we will develop general equation to find optimal flows for networks with heterogeneous QoS capabilities.

Acknowledgments. This work was supported in part by the MIC, Korea under the ITRC program supervised by the IITA and the KOSEF under the ERC program.

\section{References}

[1] SG13, "Functional Requirements and Architecture of the NG",ITU-T,May 2004.

[2] SG13, "Functional Requirements and Architecture for Resource and Admission Control in Next Generation Networks", ITU-T, May 2004.

[3] D. Xuan, C. Li, R. Bettati, J. Chen, W. Zhao, "Utilization- Based Admission Control for Real-Time Applications," The IEEE International Conference on Parallel Processing, Canada, Aug. 2000.

[4] L. Zhang, S. Deering, D. Estrin, S. Shenker and D. Zappala,"RSVP: a new resource reservation protocol," IEEENetworks Magazine, vol. 31, No. 9, pp. 8-18, September 1993.

[5] G. Papaioannou, S. Sartzetakis, and G.D. Stamoulis. Efficient agent-based selection of DiffServ SLAs over MPLS networks within the ASP service model. Journal of Network and Systems Management, Special Issue on Management of Converged Networks, Spring 2002.

[6] SG13, "An end-to-end QoS architecture based on centralized resource control for IP networks supporting NGN services", ITU-T, May 2004.

[7] SG13, "Network Performance Objectives for IP-Based Services", ITU-T, Feb. 2003.

[8] B. Teitelbaum, "QBone Architecture (vl.O)", Internet2 QoS Working Group Draft, http:iiww.inlemet2 .eduiqosiwglpapersiqbArchi I .Oidrafti2-qbone-arch-1 .O.html, Aug. 1999.

[9] S. Wang, D. Xuan, R. Bettati and W. Zhao, Providing Absolute Differentiated Services for Real-Time Applications in Static Priority Scheduling Networks, Proceedings of IEEE INFOCOM'01, April, 2001.

[10] Jamin, P. B. Danzig, S. J. Shenker, and L. Zhang. "A Measurement-based Admission Control Algorithm or Integrated Services Packet Networks (Extended Version)". ACM/IEEE Transactions on Networking, Feb. 1997. 